\title{
POINTWISE APPROXIMATION OF CORNER SINGULARITIES FOR A SINGULARLY PERTURBED REACTION-DIFFUSION EQUATION IN AN $L$-SHAPED DOMAIN
}

\author{
VLADIMIR B. ANDREEV AND NATALIA KOPTEVA
}

\begin{abstract}
A singularly perturbed reaction-diffusion equation is posed in a two-dimensional $L$-shaped domain $\Omega$ subject to a continuous Dirchlet boundary condition. Its solutions are in the Hölder space $C^{2 / 3}(\bar{\Omega})$ and typically exhibit boundary layers and corner singularities. The problem is discretized on a tensor-product Shishkin mesh that is further refined in a neighboorhood of the vertex of angle $3 \pi / 2$. We establish almost second-order convergence of our numerical method in the discrete maximum norm, uniformly in the small diffusion parameter. Numerical results are presented that support our theoretical error estimate.
\end{abstract}

\section{INTRODUCTION}

Solutions of singularly perturbed differential equations, in which highest-order derivatives are multiplied by a small parameter, typically exhibit sharp boundary and interior layers, which are narrow regions where solutions change rapidly. Furthermore, if a problem is posed in a non-smooth two-dimensional domain, solutions also exhibit corner singularities, which, being governed by the angle of the corner (similarly to standard differential equations), also involve the small parameter, and often in a non-trivial way; see, e.g., 8. Recall that corner singularities occur if special compatibility conditions induced by the corners of the domain are violated, while if the corner has angle different from $\pi / n$, for any integer $n$, compatibility conditions are non-local, i.e., they cannot be verified a priori [7, 20]. This is the case even for a simple $L$-shaped domain $\Omega$, in which one of the corners has angle $3 \pi / 2$, which results in solutions being only in the Hölder space $C^{2 / 3}(\bar{\Omega})$.

In recent years much attention has been focused on robust numerical methods for singularly perturbed problems; see, e.g., 14] for an overview; in particular, many reliable numerical solutions have been obtained in an efficient way using locally refined meshes. However, these analyses were developed mostly under the demanding assumption that exact solutions are sufficiently smooth, e.g., in $C^{k}(\bar{\Omega})$ for $k \geq 3$.

The aim or the present paper is to suggest a robust numerical method based on a suitable mesh refinement, for one singularly perturbed problem, and establish its almost second-order convergence in the discrete maximum norm, uniformly in

Received by the editor April 27, 2007 and, in revised form, August 31, 2007.

2000 Mathematics Subject Classification. Primary 65N06, 65N15, 65N50; Secondary 35B25.

Key words and phrases. Reaction-diffusion, singular perturbation, corner singularity, $L$-shaped domain, pointwise error estimate, Shishkin mesh, second order.

This research was supported by Enterprise Ireland International Collaboration Programme grant IC/2006/8. 


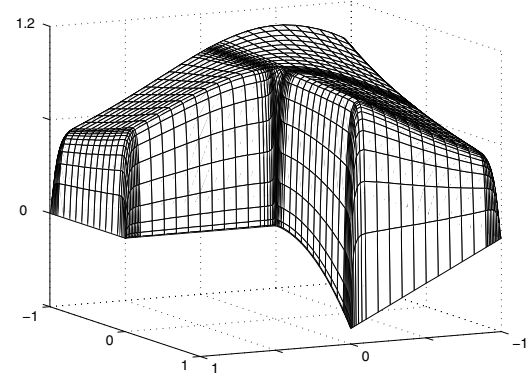

Figure 1. Solution of model problem (1.1), (6.1); $\varepsilon=0.02$.

the small parameter of the differential equation, under no demanding compatibility conditions and hence under no unrealistic assumption that exact solutions are sufficiently smooth at the corners of the domain.

Consider a singularly perturbed reaction-diffusion equation posed in a twodimensional $L$-shaped domain $\Omega=(-1,1)^{2} \backslash[0,1]^{2}$ subject to a Dirichlet boundary condition:

$$
\begin{aligned}
L u:=-\varepsilon^{2} \triangle u+q(x, y) u & =f(x, y), & & (x, y) \in \Omega, \\
u(x, y) & =g(x, y), & & (x, y) \in \partial \Omega .
\end{aligned}
$$

Here the diffusion coefficient $\varepsilon^{2} \in(0,1]$ can take arbitrarily small positive values, $\triangle=\partial^{2} / \partial x^{2}+\partial^{2} / \partial y^{2}$ is the Laplace operator, the functions $q$ and $f$ are sufficiently smooth, and

$$
q(x, y) \geq 2 \alpha^{2}=\text { const }>0 .
$$

Furthermore, $g \in C(\partial \Omega)$ and is sufficiently smooth on the sides of $\partial \Omega$.

Then problem (1.1) has a unique solution, which exhibits sharp boundary layers of width $O(\varepsilon|\ln \varepsilon|)$ along the boundary $\partial \Omega$; see Figure 1. Furthermore, the solution has corner singularities at the vertices of $\Omega$. In particular, if we introduce the standard polar coordinates $(r, \varphi)$ at the vertex $(0,0)$ of interior angle $3 \pi / 2$, then for $r \leq \varepsilon$ we have $u=C(r / \varepsilon)^{2 / 3} \sin [2(\varphi-\pi / 2) / 3]+$ smoother terms.

Our precise assumptions on $q, f$ and $g$ are as follows:

$$
q, f \in C^{4, \lambda}(\bar{\Omega}), \quad g \in C(\partial \Omega), \quad g_{k} \in C^{4, \lambda}\left(\Gamma_{k}\right), \quad k=1, \ldots, 6,
$$

for some $\lambda \in(0,1)$. Here $\left\{\Gamma_{k}\right\}_{k=1}^{6}$ are the sides of $\Omega$, ordered in the counterclockwise direction starting from $\Gamma_{1}:=\{(x, 0) \in \partial \Omega \mid x \in[0,1]\}$; see Figure 2 (left); while $g_{k}(x, y):=g(x, y)$ for $(x, y) \in \Gamma_{k}$. Furthermore, let $p_{k}, k=1, \ldots, 6$, denote the vertex that joins $\Gamma_{k}$ and $\Gamma_{k+1}$.

Note that if the boundary $\partial \Omega$ were sufficiently smooth, then the assumption of $q, f \in C^{2, \lambda}(\bar{\Omega})$ and $g \in C^{4, \lambda}(\partial \Omega)$ would imply that $u \in C^{4, \lambda}(\bar{\Omega})$ [12, Chap. 3, p. 110, (1.11)]. Since our domain has corners of angle $\pi / 2$ and $3 \pi / 2$, we only have $u \in C^{2 / 3}(\bar{\Omega}) \cap C^{4, \lambda}(\Omega)$. Note also that to get this assertion, it would suffice to assume that $q, f \in C^{2, \lambda}(\bar{\Omega})$ (compare with (1.3) $)$. Additional smoothness of $q$ and $f$ in (1.3) is required by Lemma 2.1 .

We discretize problem (1.1) using the standard second-order five-point difference scheme (see (3.1) for details) on a tensor-product Shishkin mesh [18, 19] that is 

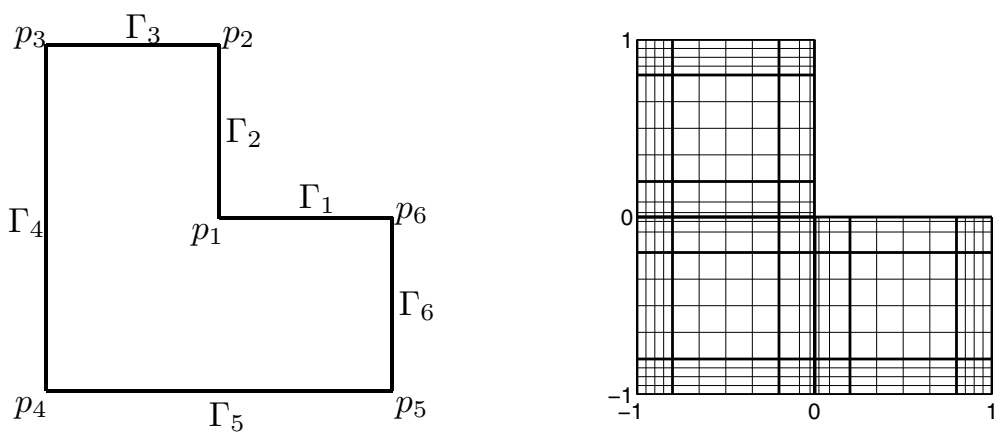

Figure 2. Domain $\Omega=(-1,1)^{2} \backslash[0,1]^{2}$ (left); mesh for $N=4$ (right).

further refined in a neighboorhood of the vertex of angle $3 \pi / 2$. Our tensor-product mesh is $\bar{\Omega}^{h}=\left\{\left(x_{i}, y_{j}\right) \in \bar{\Omega}\right\}$, where $\left\{x_{i}\right\}_{i=-3 N}^{3 N}$ and $\left\{y_{j}\right\}_{j=-3 N}^{3 N}$, such that $-1=$ $x_{-3 N}<\ldots<x_{3 N-1}<x_{3 N}=1$ and $y_{j}=x_{j}$, are two equal one-dimensional meshes on $[-1,1]$ defined as follows. Set

$$
\sigma=\min \{(2 / \alpha) \varepsilon \ln N ; 1 / 3\} .
$$

Divide each of the intervals $[-1,-1+\sigma],[-1+\sigma,-\sigma],[\sigma, 1-\sigma]$ and $[1-\sigma, 1]$ into $N$ equidistant subintervals respectively. In practice one usually has $\sigma \ll 1$ so the mesh is fine on $[-1,-1+\sigma]$ and $[1-\sigma, 1]$ and coarse on $[-1+\sigma,-\sigma]$ and $[\sigma, 1-\sigma]$.

If one uses a standard Shishkin mesh, i.e., divides the remaining part $[-\sigma, \sigma]$ of $[-1,1]$ into $2 N$ equidistant subintervals, this will yield significant errors in the neighbourhood of the vertex $p_{1}$ of angle $3 \pi / 2$, where our solution exhibits a corner singularity; similar loss of accuracy occurs even for equations that are not singularly perturbed [1].

Therefore, to resolve the corner singularity at $p_{1}$, we introduce a geometrically refined mesh $\left\{x_{i}\right\}_{i=-N}^{N}$ on $[-\sigma, \sigma]$ :

$$
x_{i}:=\sigma(i / N)^{3}, \quad i=-N, \ldots, N \text {; }
$$

see Figure 2 (right) for an example of the tensor-product mesh obtained.

Our main result, presented by Theorem 3.1, is that the error of our numerical method is $O\left(N^{-2} \ln ^{2} N\right)$ in the discrete maximum norm, uniformly in the small parameter $\varepsilon$.

Problem (1.1), (1.2), posed in various domains, has often been addressed in the numerical analysis literature. The best result known in the case of an $L$-shaped domain is $\varepsilon$-uniform convergence of order almost $2 / 11$ in the discrete maximum norm by Shishkin [19].

We also refer the reader to papers 2, 3, 5, which present maximum norm error estimates for finite difference approximations of problem (1.1), (1.2) posed in the unit square. Clavero et al. [5] argue under the assumption that the compatibility conditions of up to second order are satisfied at the corners of the domain, which, combined with an analogue of our assumption (1.3), yields $u \in C^{4, \lambda}(\bar{\Omega})$; it is proved then that the error on a Shishkin mesh is $O\left(N^{-2} \ln ^{2} N\right)$ uniformly in $\varepsilon$. Andreev [2, 3] drops the unrealistic compatibility conditions assumption and proves the same error estimate for the same numerical method assuming only an analogue of condition (1.3), i.e., when the exact solution $u$ is only in $C^{1, \lambda}(\bar{\Omega})$. Note that 
[3] also addressed the case of Dirichlet-Neumann boundary conditions and thus $u$ being only in $C^{\lambda}(\bar{\Omega})$.

Furthermore, problems similar to (1.1), (1.2) were considered in [4, 10, 13, 17. Schatz and Wahlbin [17] derive pointwise error estimates for the Galerkin finite elements on quasiuniform unrefined meshes in polygonal domains. Blatov [4 and more recently Kopteva [10] establish second-order convergence, in the discrete maximum norm, on layer-adapted meshes in a smooth domain. Melenk [13] gives an energy-norm exponential-convergence result for $h p$-finite element methods applied to a more general reaction-diffusion equation posed in a curvilinear polygon. The mesh in [13] also uses high-aspect-ratio mesh elements along the boundary and a geometric mesh refinement at the corners.

The present paper is organized as follows. In $\$ 2$ we decompose the exact solution of problem (1.1) and estimate the components of this decomposition. Next, in $\$ 3$ we describe our numerical method, state the main result and outline its proof. The entire $\$ 4$ and $\$ 5$ address the error away from the vertex $p_{1}$ of interior angle $3 \pi / 2$ and in a neighbourhood of this vertex, respectively. Finally, in 96 . numerical results are presented, which support our theoretical estimate.

Notation. Let $k$ be a non-negative integer, and $\lambda \in(0,1)$. We use the standard spaces $C^{k}(\bar{\Omega})$ of functions whose derivatives up to order $k$ are continuous in $\bar{\Omega}$, and $C^{k, \lambda}(\bar{\Omega})$, or $C^{\lambda}(\bar{\Omega})$ when $k=0$, of Hölder continuous functions in $\bar{\Omega}$. Throughout the paper, we use the notation $v^{(k, m)}:=\frac{\partial^{k+m} v}{\partial x^{m} \partial y^{m}}$ for any sufficiently smooth function $v(x, y)$. Furthermore, we let $C$ denote a generic positive constant that may take different values in different formulas, but is always independent of the mesh and $\varepsilon$. A subscripted $C$ (e.g., $C_{1}$ ) denotes a positive constant that is independent of $N$ and $\varepsilon$ and takes a fixed value. Notation such as $v=O(w)$ means $|v| \leq C w$ for some $C$.

\section{Exact solution: Decomposition And a PRiori estimates}

Lemma 2.1. Under assumption (1.3), there exists a function $v \in C^{4, \lambda}(\bar{\Omega})$ such that $L v=f$ for $(x, y) \in \Omega$ and $\left|v^{(k, m)}\right| \leq C\left[1+\varepsilon^{2-k-m}\right]$.

Proof. We imitate the argument used in [5, §2]. Let $\Omega^{*}:=(-2,2) \times(-2,2)$. Define smooth extensions $q^{*}, f^{*} \in C^{4, \lambda}\left(\bar{\Omega}^{*}\right)$ of $q, f \in C^{4, \lambda}(\bar{\Omega})$ so that $q^{*}=q$ and $f^{*}=f$ in $\bar{\Omega}$. Furthermore, introduce a smooth boundary condition $g^{*}$ on $\partial \Omega^{*}$ such that the solution $v^{*}$ of the equation $L^{*} v^{*}=f^{*}$, subject to the boundary condition $v^{*}=g^{*}$ on $\partial \Omega^{*}$, is in $C^{4, \lambda}\left(\bar{\Omega}^{*}\right)$. Here the operator $L^{*}$ is defined similarly to $L$ in (1.1) with $q$ replaced by $q^{*}$. Then [5, (2.5)] the function $v:=v^{*}$ in $\bar{\Omega}$ satisfies the assertion of our lemma.

It is crucial in the above argument that any function in $C^{4, \lambda}(\bar{\Omega})$ can be extended to $C^{4, \lambda}\left(\bar{\Omega}^{*}\right)$, if $\Omega$ is an $L$-shaped domain [6, $\left.\S 261\right]$.

Now we decompose the solution $u$ of problem (1.1) as $u=v+w$, where $L w=0$ and the component $w$ describes the boundary layers of width $O(\varepsilon|\ln \varepsilon|)$ along each of the six sides and corner singularities at each of the six vertices of our domain $\Omega$. Note that the boundary layers along the sides $\Gamma_{k}, k=1, \ldots, 6$, and the corner singularities at the vertices $p_{k}, k=2, \ldots, 6$, of angle $\pi / 2$ are typical phenomena in the case of a rectangular domain; both their analytical and numerical analyses are presented in [5, 2, 3]. To simplify our presentation, we shall focus on the corner singularity at the vertex $p_{1}$ of interior angle $3 \pi / 2$ and hence make the following assumption. 
Assumption A. Let the boundary condition function $g$ from (1.1) satisfy $g=v$ on $\partial \Omega \backslash\left(\tilde{\Gamma}_{1} \cup \tilde{\Gamma}_{2}\right)$, where $v$ is from Lemma 2.1 and $\tilde{\Gamma}_{1}:=\left\{(x, 0) \mid x \in\left[0,1-C_{0}\right]\right\} \subset \Gamma_{1}$, $\tilde{\Gamma}_{2}:=\left\{(0, y) \mid y \in\left[0,1-C_{0}\right]\right\} \subset \Gamma_{2}$ for some constant $C_{0}<1$.

Remark 2.2. Note that Assumption A is made only to simplify the presentation. Combining the analysis that we present under Assumption A with the analyses [2, 3] for the rectangular domain, yields our main result, Theorem [3.1, without Assumption A.

Remark 2.3. Assumption A implies that the compatibility conditions at the vertices $p_{k}, k=2, \ldots, 6$, of up to second order are satisfied so that $u \in C^{4, \lambda}\left(\bar{\Omega} \backslash p_{1}\right)$.

Theorem 2.4. (i) Under Assumption A, the solution $u$ of problem (1.1), (1.2), (1.3) satisfies

$$
\left|u^{(k, m)}\right| \leq C \varepsilon^{-k-m}\left[1+(r / \varepsilon)^{2 / 3-k-m}\right], \quad 1 \leq k+m \leq 4,
$$

where $r:=\sqrt{x^{2}+y^{2}}$. In particular, for $r \leq \varepsilon$ we have $u=\chi+z$, where

$$
|\chi| \leq C(r / \varepsilon)^{2 / 3}, \quad\left|z^{(k, m)}\right| \leq C \varepsilon^{-k-m} .
$$

(ii) Furthermore, $u$ allows the decomposition $u=v+w$ in $\bar{\Omega}$, where $v$ is from Lemma 2.1 and

$$
\left|v^{(k, m)}\right| \leq C\left[1+\varepsilon^{2-k-m}\right], \quad|w| \leq C \min \left\{e^{\alpha x / \varepsilon} ; e^{\alpha y / \varepsilon}\right\} ;
$$

and there exist functions $w_{1}$ and $w_{2}$ defined in the domains $\Omega_{1}:=(0,1) \times(-1,0)$ and $\Omega_{2}:=(-1,0) \times(0,1)$, respectively, such that

$$
L w_{1}=0, \quad\left|w-w_{1}\right| \leq C e^{-\alpha x / \varepsilon}, \quad\left|w_{1}^{(k, m)}\right| \leq C\left[1+\varepsilon^{2-k}+\varepsilon^{-m}\right] \quad \text { in } \Omega_{1} ;
$$

$$
L w_{2}=0, \quad\left|w-w_{2}\right| \leq C e^{-\alpha y / \varepsilon}, \quad\left|w_{2}^{(k, m)}\right| \leq C\left[1+\varepsilon^{-k}+\varepsilon^{2-m}\right] \quad \text { in } \Omega_{2} ;
$$

for $0 \leq k+m \leq 4$.

Proof. (i) Since the operator $L$ satisfies the maximum/comparison principle, we have $|u| \leq C$. Let the stretching transformation from $(x, y)$ to the new coordinates $\hat{x}:=x / \varepsilon$ and $\hat{y}:=y / \varepsilon$ map the original domain $\Omega$ into the domain $\hat{\Omega}$, in which (1.1) implies $-\triangle \hat{u}+\hat{q} \hat{u}=\hat{f}$, where the notation $\hat{v}(\hat{x}, \hat{y}):=v(x, y)$ is used for any function $v$. Next, restrict $\hat{u}$ to the subdomain $\hat{\Omega}^{\prime}$, which is obtained from $\hat{\Omega}$ by excluding such $O(1)$ neighbourhoods of each of its six vertices that $\partial \hat{\Omega}^{\prime}$ is sufficiently smooth. Then, using Schauder-type estimates (see, e.g., 12, Chap. 3, p.110, (1.13)]) one can show that $\left|\hat{u}^{(k, m)}\right| \leq C$ in $\hat{\Omega}^{\prime}$. This implies (2.1a) in the corresponding subdomain $\Omega^{\prime}$ of $\Omega$ since $r \geq C \varepsilon$ in $\Omega^{\prime}$. Thus it remains to obtain (2.1) in a certain $O(\varepsilon)$ neighbourhood of each of the six vertices of $\Omega$.

Next consider the $O(\varepsilon)$ neighbourhood of any of the vertices $p_{j}, j=2, \ldots, 6$ of interior angle $\pi / 2$, e.g., $p_{4}=(-1,-1)$. This neighbourhood is covered by the rectangle $D:=(-1,-1 / 2)^{2}$, on whose boundary $u \in C^{4, \lambda}$, and at whose vertices the compatibility conditions are satisfied by Remark 2.3. Hence estimate (2.1a) holds true in $\bar{D}$ by [5, Theorem 2.2].

Finally, consider the $\varepsilon$-neighbourhood of the vertex $p_{1}$ of interior angle $3 \pi / 2$, which can be covered by a domain $\Omega_{\varepsilon} \subset(-C \varepsilon, C \varepsilon)^{2} \cap \Omega$ such that $\partial \Omega_{\varepsilon} \backslash p_{1}$ is smooth. Then, by Remark 2.3 , we have $u \in C^{4, \lambda}\left(\partial \Omega_{\varepsilon} \backslash p_{1}\right)$. Next, transforming $\Omega_{\varepsilon}$ 
by $(\hat{x}, \hat{y})=(x, y) / \varepsilon$ into $\hat{\Omega}_{\varepsilon}$, we get $\hat{L} \hat{u}=-\triangle \hat{u}+\hat{q} \hat{u}=\hat{f}$ in $\hat{\Omega}_{\varepsilon}$, with $\hat{u}=\hat{g}$ on $\partial \hat{\Omega}_{\varepsilon} \cap \partial \hat{\Omega}$ and $\left|\hat{u}^{(k, m)}\right| \leq C$ on the remaining part of $\partial \hat{\Omega}_{\varepsilon}$. To establish (2.1) in $\bar{\Omega}_{\varepsilon}$, it suffices to show that in the domain $\hat{\Omega}_{\varepsilon}$ we have

$$
\hat{u}=\hat{\chi}+\hat{z}, \quad\left|\hat{\chi}^{(m, k)}\right| \leq C \hat{r}^{2 / 3-m-k}, \quad\left|\hat{z}^{(m, k)}\right| \leq C \quad \text { for } 0 \leq k+m \leq 4,
$$

where $\hat{r}=\sqrt{\hat{x}^{2}+\hat{y}^{2}}$. Note that the above decomposition in the case of $\hat{q} \equiv 0$ immediately follows from [7, Theorem 6.4.2.6]. Our operator $\hat{L}$ involves $\hat{q}>0$ and thus is not directly covered by the analysis in 7 . We also refer the reader to the classical technique by Kondrat'ev [9, which yields decompositions similar to (2.3) for general elliptic operators, with the smooth-component analogue of $\hat{z}$ being in a suitable Sobolev space, while we require $\hat{z}$ to be in the Hölder space $C^{4, \lambda}$. Hence we shall outline a proof of (2.3) for $\hat{L}$ with $\hat{q}>0$; see [8, p. 182] for a similar argument.

First, note that $\hat{u} \in C$ implies $\triangle \hat{u} \in L_{2}$, which, by [7, Theorem 5.1.3.5], yields $\hat{u}=\hat{\chi}_{0}+\hat{z}_{0}$, where $\hat{\chi}_{0}$ is a singular function such that $\left|\hat{\chi}_{0}\right| \leq C \hat{r}^{2 / 3}$ and $\hat{\chi}_{0} \in C^{2 / 3}$, while $\hat{z}_{0} \in W_{2}^{2}$. Here $W_{2}^{2}$ is a standard Sobolev space, for which have $W_{2}^{2} \subset C^{2 / 3}$ by the Sobolev imbedding theorem; see, e.g., [7, §1.4.4]. Thus $\hat{u} \in C^{2 / 3}$ and hence $\triangle \hat{u} \in C^{2 / 3}$. Now, [7, Theorem 6.4.2.6] yields $\hat{u}=\hat{\chi}_{1}+\hat{z}_{1}$, where $\hat{\chi}_{1}$ is a singular function similar to $\hat{\chi}_{0}$, while $\hat{z}_{1} \in C^{2,2 / 3}$. Finally, we decompose as $\hat{u}=\hat{\chi}_{2}+\hat{z}_{2}$, where $\triangle \hat{\chi}_{2}=\hat{q} \hat{\chi}_{1}$ and $\triangle \hat{z}_{2}=\hat{q} \hat{z}_{1}-\hat{f}$. Then applying [7, Theorem 6.4.2.6] again we see that $\hat{z}_{2}=\hat{\chi}_{3}+\hat{z}$ with $\hat{z} \in C^{4, \lambda^{\prime}}, \lambda^{\prime}=\min \{2 / 3, \lambda\}$. Furthermore, $\hat{\chi}:=\hat{\chi}_{2}+\hat{\chi}_{3}$ is a singular function that satisfies (2.3), where $\hat{\chi}_{2}$ is estimated using [9, Theorem 1.3]. Thus we established (2.3) in $\hat{\Omega}_{\varepsilon}$.

(ii) The estimate for $v$ in (2.2a) immediately follows from Lemma 2.1, Next, combining (1.1) with $L v=f$ and Assumption A, we arrive at the following problem for $w=u-v$ :

$$
L w=0 \text { in } \Omega ; \quad w=g-v \text { on } \tilde{\Gamma}_{1} \cup \tilde{\Gamma}_{2} ; \quad w=0 \text { on } \partial \Omega \backslash\left(\tilde{\Gamma}_{1} \cup \tilde{\Gamma}_{2}\right) .
$$

By the maximum/comparison principle, $|w| \leq C$ in $\bar{\Omega}$. Now, restrict $w$ to the rectangle $[-1,0] \times[-1,1]$, on whose boundary $w=0$ except for the side $x=0$, where $|w| \leq C$. Applying the comparison principle, we see that $|w| \leq C e^{\alpha x / \varepsilon}$ in this rectangle. Combining this with a similar estimate $|w| \leq C e^{\alpha y / \varepsilon}$ in $[-1,1] \times[-1,0]$, we get the desired estimate for $w$ in (2.2a).

It remains to obtain (2.2b) as estimate (2.2c) is similar. Introduce the domain $\Omega_{1}^{* *}:=(-1,1) \times(-1,0)$ and extend the boundary condition function $g-v$ to $\{(x, 0) \mid x \in[-1,0)\}$ so that $[g-v]^{* *}(x, 0)=0$ for $x \in[-1,-1 / 2]$. Now, define $w_{1}:=w_{1}^{*}$ in $\bar{\Omega}_{1}$, where $w_{1}^{*}$ is a solution to the following problem:

$$
L w_{1}^{*}=0 \text { in } \Omega_{1}^{* *}, \quad w_{1}^{*}(-1, y)=w_{1}^{*}(1, y)=w_{1}^{*}(x,-1)=0, \quad w_{1}^{*}(x, 0)=[g-v]^{* *} ;
$$

compare with [5, problem (2.6)]. Imitating the argument used in [5, pp. 1746-1747], we get $\left|w_{1}^{(k, m)}\right| \leq C\left[1+\varepsilon^{2-k}+\varepsilon^{-m}\right]$. Finally, by the comparison principle, the estimate $\left|w-w_{1}\right| \leq C e^{-\alpha x / \varepsilon}$ follows from $L\left[w-w_{1}\right]=0$ in $\Omega_{1}$ and $w=w_{1}$ on $\partial \Omega_{1}$ except for the side $x=0$, where $\left|w-w_{1}\right| \leq C$.

\section{Numerical method. Main Result}

We require the computed solution $U$ to satisfy the standard five-point finitedifference discretization of problem (1.1):

$$
\begin{aligned}
L^{h} U_{i j}:=-\varepsilon^{2} \triangle^{h} U_{i j}+q\left(x_{i}, y_{j}\right) U_{i j} & =f\left(x_{i}, y_{j}\right), & \left(x_{i}, y_{j}\right) \in \Omega^{h}, \\
U_{i j} & =g\left(x_{i}, y_{j}\right), & \left(x_{i}, y_{j}\right) \in \partial \Omega^{h},
\end{aligned}
$$


where

$$
\triangle^{h} U_{i j}:=\frac{\frac{U_{i+1, j}-U_{i j}}{h_{i+1}}-\frac{U_{i j}-U_{i-1, j}}{h_{i}}}{\hbar_{i}}+\frac{\frac{U_{i, j+1}-U_{i j}}{h_{j+1}}-\frac{U_{i j}-U_{i, j-1}}{h_{j}}}{\hbar_{j}} .
$$

Here, as usual, $U_{i j}$ is associated with the mesh node $\left(x_{i}, y_{j}\right)$ of the tensor-product mesh $\bar{\Omega}^{h}$ described in $₫ 1$, while $\partial \Omega^{h}:=\partial \Omega \cap \bar{\Omega}^{h}$ and $\Omega^{h}:=\Omega \cap \bar{\Omega}^{h}$. In (3.1c) we used the standard notation $h_{i}:=x_{i}-x_{i-1}=y_{i}-y_{i-1}$ and $\hbar_{i}=\left(h_{i}+h_{i+1}\right) / 2$. One can easily check that $h_{i} \leq C N^{-1}$ for all $i$. In particular, for $i=1, \ldots, N$, by (1.5), we have $h_{i}=h_{-i+1}=x_{i}-x_{i-1}=\sigma\left(3 i^{2}-3 i+1\right) / N^{3}$, which implies

$$
h_{i} \leq C N^{-1}, \quad i=-N+1, \ldots, N ; \quad\left|h_{i+1}-h_{i}\right| \leq C N^{-2}, \quad i=-N+1, \ldots, N-1 .
$$

Note that, by condition (1.2), the discrete operator $L^{h}$ from (3.1a) satisfies the discrete maximum/comparison principle, and furthermore, there exists a unique solution of discrete problem (3.1) [15, 16.

Now we state our main result.

Theorem 3.1. Let $u(x, y)$ be a solution of problem (1.1), (1.2), (1.3), and $U_{i j}$ a solution of discrete problem (3.1). Then

$$
\left|U_{i j}-u\left(x_{i}, y_{j}\right)\right| \leq C N^{-2} \ln ^{2} N, \quad\left(x_{i}, y_{j}\right) \in \bar{\Omega}^{h} .
$$

Proof. To simplify the presentation, we give a proof invoking Theorem 2.4 i.e., under the simplifying Assumption A that we made in $§ 2$. Note that one can establish the general case of Theorem 3.1, without Assumption A; see Remark 2.2 .

Consider two cases.

Case A. $\sigma<1 / 3$. In this case, the proof is made in two steps, to which we devote all of $\S \S 4$, 5. First, in $\$ 4$, the error estimate (3.3) is obtained in the subdomain $\bar{\Omega}^{h} \backslash(-\sigma, \sigma)^{2}$; then in $\$ 5$ we prove (3.3) in the remaining neighbourhood of the vertex $p_{1}$ of interior angle $3 \pi / 2$. Combining Corollary 4.4 and Theorem 5.2 , yields the desired estimate (3.3) in Case A.

Case B. $\sigma=1 / 3$. Then by (1.4), we have $\varepsilon^{-1} \leq C \ln N$. Since this case is less interesting, we shall only sketch the proof. Transforming the domain $\Omega$ by $(\hat{x}, \hat{y})=$ $(x, y) / \varepsilon$ into $\hat{\Omega}$, we obtain a problem in $\hat{\Omega}$ similar to (1.1), but with $\varepsilon:=1$. Our mesh $\Omega^{h}$ is then transformed into the mesh $\hat{\Omega}^{h}$ with $h_{i} \leq C \varepsilon^{-1} N^{-1} \leq C N^{-1} \ln N$. Now, the proof is made in a single step, which applies to the whole domain $\hat{\Omega}$ and combines the arguments used to prove Lemma 4.2 and Theorem 5.2

\section{ERror AWAY From the Vertex of ANGLE $3 \pi / 2$}

In this section we shall estimate the error $U_{i j}-u\left(x_{i}, y_{j}\right)$ in $\bar{\Omega}^{h} \backslash(-\sigma, \sigma)^{2}$, i.e., at a distance of $O(\sigma)$ away from the vertex $p_{1}$ of interior angle $3 \pi / 2$.

Modifying a barrier function from [5], introduce the auxiliary discrete function $B\left(x_{i_{0}} ; x_{i}\right)$ defined by

$$
B\left(x_{i_{0}} ; x_{i}\right):=\left\{\begin{array}{cc}
1, & x_{i} \geq x_{i_{0}}, \\
\prod_{j=i+1}^{i_{0}}\left(1+\alpha h_{j} / \varepsilon\right)^{-1}, & x_{i}<x_{i_{0}} .
\end{array}\right.
$$

This function is a discrete analogue of $\min \left\{e^{-\alpha\left(x_{i_{0}}-x\right)} ; 1\right\}$. 
Lemma 4.1. For the function $B\left(x_{i_{0}} ; x_{i}\right)$ from (4.1), we have

$$
\begin{gathered}
L^{h} B\left(x_{i_{0}} ; x_{i}\right) \geq\left\{\begin{array}{cc}
0, & x_{i} \neq x_{i_{0}}, \\
\alpha \varepsilon N, & x_{i}=x_{i_{0}}
\end{array}\right. \\
L^{h} B\left(0 ;-x_{i}\right) \geq 0, \quad x_{i}>0 .
\end{gathered}
$$

Furthermore,

$$
B(0 ;-\sigma) \leq C N^{-2} \quad \text { if } \quad \sigma<1 / 3 .
$$

Proof. To simplify the presentation, we fix $i_{0}$ and use the notation $B_{i}:=B\left(x_{i_{0}} ; x_{i}\right)$ throughout this proof. The estimate $L^{h} B_{i} \geq 0$ in (4.2a) is straightforward for $i>i_{0}$, while for $i<i_{0}$, a similar estimate is obtained in [5, (3.10)]. Thus it remains to consider $L^{h} B_{i_{0}}$. Since $D^{-} B_{i}=(\alpha / \varepsilon) B_{i-1}$ for $i \leq i_{0}$, a calculation shows that

$$
L^{h} B_{i_{0}}=\frac{\alpha \varepsilon}{\hbar_{i_{0}}} B_{i_{0}-1}+q\left(x_{i_{0}}, y_{j}\right) \geq \frac{\alpha \varepsilon}{\hbar_{i_{0}}}(1+t)^{-1}+\frac{2 \alpha \varepsilon}{h_{i_{0}}} t, \quad t:=\frac{\alpha h_{i_{0}}}{\varepsilon},
$$

where we invoked (1.2). Now, noting that $\hbar_{i_{0}}$ and $h_{i_{0}}$ do not exceed $N^{-1}$, while $(1+t)^{-1}+2 t \geq 1 \quad \forall t$, we obtain (4.2a) for $x_{i}=x_{i_{0}}$.

Estimate (4.2b) is similar to (4.2a) for $i<i_{0}$.

Finally, estimate (4.3) follows from $\left(1+\alpha h_{j} / \varepsilon\right)^{-1} \leq \exp \left[-\alpha h_{j} / \varepsilon+C\left(h_{j} / \varepsilon\right)^{2}\right]$. Indeed, combining this with (1.4) and $\sigma<1 / 3$, we get

$$
B(0 ;-\sigma)=B\left(x_{0} ; x_{-N}\right) \leq e^{-\alpha \sigma / \varepsilon}\left[1+C \sum_{j=-N+1}^{0}\left(h_{j} / \varepsilon\right)^{2}\right] \leq C N^{-2} .
$$

Next, we introduce a discrete analogue $V_{i j}$ of the function $v$ from Lemma 2.1.

$$
L^{h} V_{i j}=f_{i j} \quad \text { in } \Omega^{h} ; \quad V_{i j}=v\left(x_{i}, y_{j}\right) \quad \text { on } \partial \Omega^{h} .
$$

Lemma 4.2. Let the function $v$ be from Lemma 2.1 and let $V_{i j}$ be a solution of problem (4.4). Then $\left|V_{i j}-v\left(x_{i}, y_{j}\right)\right| \leq C N^{-2}$ for $\left(x_{i}, y_{j}\right) \in \bar{\Omega}^{h}$.

Proof. Since we closely imitate the proof of [5, estimate (3.8)], we only sketch our argument. Invoking the estimate for $v$ in (2.2a), we get

$$
\left|L^{h}\left[V_{i j}-v\left(x_{i}, y_{j}\right)\right]\right| \leq C\left\{\begin{array}{cl}
\varepsilon N^{-1}, & x_{i} \text { or } y_{j} \in\{ \pm(1-\sigma), \pm \sigma\} \\
N^{-2}, & \text { otherwise. }
\end{array}\right.
$$

Note that the first line here corresponds to the transition points of our mesh, while the second line corresponds to the points, where the mesh is uniform or, if $x_{i}$ or $y_{j}$ is in $(-\sigma, \sigma)$, where the mesh is smooth and satisfies $\left|h_{i+1}-h_{i}\right| \leq C N^{-2}$, by (3.2). At the transition points $\pm(1-\sigma), \pm \sigma$, we used the calculation $\left|L^{h}\left[V_{i j}-v\left(x_{i}, y_{j}\right)\right]\right| \leq$ $C \varepsilon\left|h_{i+1}-h_{i}\right| \leq \varepsilon N^{-1}$.

Now, to estimate $V_{i j}-v\left(x_{i}, y_{j}\right)$, we recall $B\left(x_{i_{0}} ; x_{i}\right)$ defined in (4.1), construct the discrete barrier function

$$
\begin{aligned}
\mathcal{B}_{i j}:=\bar{C} N^{-2}[1 & +B\left(-1+\sigma ; x_{i}\right)+B\left(-\sigma ; x_{i}\right)+B\left(\sigma ; x_{i}\right)+B\left(1-\sigma ; x_{i}\right) \\
& \left.+B\left(-1+\sigma ; y_{j}\right)+B\left(-\sigma ; y_{j}\right)+B\left(\sigma ; y_{j}\right)+B\left(1-\sigma ; y_{j}\right)\right],
\end{aligned}
$$

and, invoking the discrete comparison principle and (4.2a), obtain $\left|V_{i j}-v\left(x_{i}, y_{j}\right)\right| \leq$ $\mathcal{B}_{i j} \leq C N^{-2}$. 
Imitating the decomposition $u=v+w$ of Theorem 2.4, we decompose our computed solution $U_{i j}$ as $U_{i j}=V_{i j}+W_{i j}$, where $W_{i j}$ is a discrete analogue of the solution $w$ of problem (2.4). Thus $W_{i j}$ satisfies

$$
L^{h} W_{i j}=0 \quad \text { in } \Omega^{h} ; \quad W_{i j}=\left\{\begin{array}{cl}
w\left(x_{i}, y_{j}\right) & \text { on } \partial \Omega^{h} \cap\left(\tilde{\Gamma}_{1} \cup \tilde{\Gamma}_{2}\right) ; \\
0 & \text { on } \partial \Omega^{h} \backslash\left(\tilde{\Gamma}_{1} \cup \tilde{\Gamma}_{2}\right) .
\end{array}\right.
$$

Lemma 4.3. Let $w$ be from Theorem 2.4, let $W_{i j}$ be a solution of problem (4.5), and let $\sigma<1 / 3$. Then

$$
\left|W_{i j}-w\left(x_{i}, y_{j}\right)\right| \leq C\left\{\begin{array}{cl}
N^{-2} & \text { in } \bar{\Omega}^{h} \backslash(-\sigma, 1]^{2}, \\
N^{-2} \ln ^{2} N & \text { in } \bar{\Omega}^{h} \cap([\sigma, 1] \times[-\sigma, 0] \cup[-\sigma, 0] \times[\sigma, 1]) .
\end{array}\right.
$$

Proof. First, we obtain the desired estimate in $\Omega^{h} \backslash(-\sigma, 1]^{2}$. Using $C B\left(0, x_{i}\right)$ and $C B\left(0, y_{j}\right)$ (recall (4.1) and (4.2a)) as barrier functions for problem (4.5), we observe that $\left|W_{i j}\right| \leq C \min \left\{B\left(0, x_{i}\right) ; B\left(0 ; y_{j}\right)\right\}$. This implies that in $\Omega^{h} \backslash(-\sigma, 1]^{2}$ we have $\left|W_{i j}\right| \leq C B(0,-\sigma) \leq C N^{-2}$, where we invoked (4.3). Now, combining (2.2a), (1.4), and $\sigma<1 / 3$, we also get $|w| \leq C e^{-\alpha \sigma / \varepsilon} \leq C N^{-2}$ in the same domain. Since both $W_{i j}$ and $w$ are $O\left(N^{-2}\right)$ in $\Omega^{h} \backslash(-\sigma, 1]^{2}$, the desired estimate follows.

It remains to prove the estimate of our lemma in $\Omega_{1}^{\prime}:=[\sigma, 1] \times[-\sigma, 0]$ as in the domain $[-\sigma, 0] \times[\sigma, 1]$ it is obtained similarly. Restrict the function $w_{1}$ of $(2.2 \mathrm{~b})$ to $\Omega_{1}^{\prime \prime}:=(0,1) \times(-\sigma, 0) \supset \Omega_{1}^{\prime}$ and let its discrete analogue $W_{1, i j}$ satisfy

$$
L^{h} W_{1, i j}=0 \quad \text { in } \bar{\Omega}^{h} \cap \Omega_{1}^{\prime \prime}, \quad W_{1, i j}=w_{1}\left(x_{i}, y_{j}\right) \quad \text { on } \bar{\Omega}^{h} \cap \partial \Omega_{1}^{\prime \prime} .
$$

Then, imitating the proof of [5. Proposition 3.1], we get $\left|W_{1}-w_{1}\right| \leq C N^{-2} \ln ^{2} N$ in $\Omega_{1}^{\prime \prime}$ and hence in $\Omega_{1}^{\prime}$. By (2.2b), we also have $\left|w-w_{1}\right| \leq C e^{-\alpha \sigma / \varepsilon} \leq C N^{-2}$ in $\Omega_{1}^{\prime}$. Thus to get the desired estimate, it suffices to show $\left|W-W_{1}\right| \leq C N^{-2}$ in $\Omega_{1}^{\prime}$. Note that $L^{h}\left[W-W_{1}\right]=0$ in $\Omega_{1}^{\prime \prime}$, while on $\partial \Omega_{1}^{\prime \prime}$ we have $\left|W-W_{1}\right|=$ $\left|(W-w)+\left(w-w_{1}\right)\right| \leq C\left[N^{-2}+e^{-\alpha x / \varepsilon}\right]$. Now invoking the comparison principle, we see that $\left|W-W_{1}\right| \leq C\left[B\left(0,-x_{i}\right)+N^{-2}\right]$ in $\Omega_{1}^{\prime \prime}$, and hence $\left|W-W_{1}\right| \leq C N^{-2}$ in $\Omega_{1}^{\prime}$.

Corollary 4.4. Let $u$ be a solution of problem (1.1), (1.2), (1.3), and $U_{i j}$ a solution of problem (3.1). Then if $\sigma<1 / 3$, we have $\left|U_{i j}-u\left(x_{i}, y_{j}\right)\right| \leq C N^{-2} \ln ^{2} N$ in $\bar{\Omega}^{h} \backslash(-\sigma, \sigma)^{2}$.

Proof. Recall that $u=v+w$ and $U_{i j}=V_{i j}+W_{i j}$. Now combine Lemma 4.3 with Lemma 4.2 .

\section{Error in a neighbourhood of the Vertex of ANgLe $3 \pi / 2$}

In this section we continue to consider the case of $\sigma<1 / 3$. It remains to estimate $U-u$ in the subdomain $\Omega_{\sigma}:=(-\sigma, \sigma)^{2} \backslash[0, \sigma]^{2}$. Since, by Corollary 4.4 we already know that $U-u=O\left(N^{-2} \ln ^{2} N\right)$ on $\partial \Omega_{\sigma}$, we restrict both $U$ and $u$ to $\bar{\Omega}_{\sigma}$.

Next, transform the domain $\Omega_{\sigma}$ by $(\hat{x}, \hat{y}):=(x, y) / \varepsilon$ into $\hat{\Omega}_{\sigma}:=(-\hat{\sigma}, \hat{\sigma})^{2} \backslash[0, \hat{\sigma}]^{2}$, where $\hat{\sigma}=\sigma / \varepsilon$ and, by (1.4) combined with $\sigma<1 / 3$, we get

$$
\hat{\sigma}:=(2 / \alpha) \ln N \text {. }
$$

Note that (1.1) implies that $\hat{L} \hat{u}:=-\triangle \hat{u}+\hat{q} \hat{u}=\hat{f}$ in $\hat{\Omega}_{\sigma}$, where the notation $\hat{v}(\hat{x}, \hat{y}):=v(x, y)$ is used for any function $v$. Furthermore, by (2.1), the function $\hat{u}$ satisfies

$$
\left|\hat{u}^{(k, m)}\right| \leq C\left[\hat{r}^{2 / 3-k-m}+1\right],
$$


where $\hat{r}:=\sqrt{\hat{x}^{2}+\hat{y}^{2}}$, and can be decomposed as

$$
\hat{u}=\hat{\chi}+\hat{z}, \quad|\hat{\chi}| \leq C \hat{r}^{2 / 3}, \quad\left|\hat{z}^{(k, m)}\right| \leq C .
$$

Now our computed solution $U_{i j}$ is associated with the mesh nodes

$$
\left(\hat{x}_{i}, \hat{y}_{j}\right):=\hat{\sigma}\left(i^{3}, j^{3}\right) / N^{3}, \quad i, j=-N, \ldots, N,
$$

(compare with (1.5)) and satisfies

$$
\hat{L}^{h} U_{i j}:=-\hat{\triangle}^{h} U_{i j}+\hat{q}\left(\hat{x}_{i}, \hat{y}_{j}\right) U_{i j}=\hat{f}\left(\hat{x}_{i}, \hat{y}_{j}\right), \quad\left(\hat{x}_{i}, \hat{y}_{j}\right) \in \hat{\Omega}_{\sigma},
$$

where the discrete operator $\hat{\triangle}^{h}$ is defined similarly to $\Delta^{h}$ from (3.1c) with the new values of $h_{i}:=\hat{x}_{i}-\hat{x}_{i-1}$ and $\hbar_{i}:=\left(\hat{x}_{i+1}-\hat{x}_{i-1}\right) / 2$. Furthermore, by (5.3), we have

$$
h_{i}=\hat{x}_{i}-\hat{x}_{i-1}=\hat{\sigma} \frac{3 i^{2}-3 i+1}{N^{3}}<\hat{\sigma} \frac{3 i^{2}}{N^{3}}, \quad h_{i+1}-h_{i}=\hat{\sigma} \frac{6 i}{N^{3}}, \quad \text { for } i>0,
$$

while $h_{1}-h_{0}=0$, and $h_{-i}=h_{i+1}, h_{-i+1}-h_{-i}=-\left(h_{i+1}-h_{i}\right)$ for $i \geq 0$.

5.1. Error estimate in $\Omega_{\sigma}$. To estimate the error in the subdomain $\Omega_{\sigma}$, we shall invoke the following auxiliary lemma.

Lemma 5.1. If a discrete function $\phi_{i j}$ satisfies

$$
\left|\hat{L}^{h} \phi_{i j}\right| \leq \hat{r}_{i j}^{-2}+1, \quad\left(\hat{x}_{i}, \hat{y}_{j}\right) \in \hat{\Omega}_{\sigma} ; \quad\left|\phi_{i j}\right| \leq C, \quad\left(\hat{x}_{i}, \hat{y}_{j}\right) \in \partial \hat{\Omega}_{\sigma},
$$

where $\hat{r}_{i j}:=\sqrt{\hat{x}_{i}^{2}+\hat{y}_{j}^{2}}$, then $\left|\phi_{i j}\right| \leq C$.

Proof. We defer the proof to $\$ 5.2$

Now we present the main result of this section.

Theorem 5.2. Let $u$ and $U_{i j}$ be solutions of problems (1.1), (1.2), (1.3) and (3.1) respectively, and let $\sigma<1 / 3$. Then $\left|U_{i j}-u\left(x_{i}, y_{j}\right)\right| \leq C N^{-2} \ln ^{2} N$ in $\Omega^{h} \cap(-\sigma, \sigma)^{2}$.

Proof. We have to estimate the error $e_{i j}:=U_{i j}-u\left(x_{i}, y_{j}\right)=U_{i j}-\hat{u}\left(\hat{x}_{i}, \hat{y}_{j}\right)$ for $\left(x_{i}, y_{j}\right) \in \Omega^{h} \cap(-\sigma, \sigma)^{2}$, i.e., for $\left(\hat{x}_{i}, \hat{y}_{j}\right) \in \hat{\Omega}_{\sigma}$. Using (5.4) and Corollary 4.4, one can easily check that

$$
\hat{L}^{h} e_{i j}=\psi_{i j}, \quad\left(\hat{x}_{i}, \hat{y}_{j}\right) \in \hat{\Omega}_{\sigma} ; \quad\left|e_{i j}\right| \leq C N^{-2} \ln ^{2} N, \quad\left(\hat{x}_{i}, \hat{y}_{j}\right) \in \partial \hat{\Omega}_{\sigma},
$$

where

$$
\psi_{i j}:=\hat{\triangle}^{h} \hat{u}\left(\hat{x}_{i}, \hat{y}_{j}\right)-\triangle \hat{u}\left(\hat{x}_{i}, \hat{y}_{j}\right)
$$

is the truncation error. We claim that

$$
\left|\psi_{i j}\right| \leq C N^{-2} \ln ^{2} N\left(\hat{r}_{i j}^{-2}+1\right), \quad\left(\hat{x}_{i}, \hat{y}_{j}\right) \in \hat{\Omega}_{\sigma} .
$$

Then, applying the discrete maximum/comparison principle to problem (5.7), we obtain $\left|e_{i j}\right| \leq C\left(N^{-2} \ln ^{2} N\right) \phi_{i j}$, where $\phi_{i j}$ is from Lemma 5.1. This implies the desired error estimate.

Thus, to complete the proof, it suffices to show (5.9). Consider two cases.

Case A. $|i|+|j| \geq 2$. Using Taylor series expansions, we get $\psi_{i j}=\psi_{1, i j}+\psi_{2, i j}$, where

$$
\begin{aligned}
& \left|\psi_{1, i j}\right| \leq C\left(\left|\left(h_{i+1}-h_{i}\right) \hat{u}^{(3,0)}\left(\hat{x}_{i}, \hat{y}_{j}\right)\right|+\hbar_{i}^{2}\left|\hat{u}^{(4,0)}\left(\hat{\xi}_{i j}, \hat{y}_{j}\right)\right|\right), \\
& \left|\psi_{2, i j}\right| \leq C\left(\left|\left(h_{j+1}-h_{j}\right) \hat{u}^{(0,3)}\left(\hat{x}_{i}, \hat{y}_{j}\right)\right|+\hbar_{j}^{2}\left|\hat{u}^{(0,4)}\left(\hat{x}_{i}, \hat{\eta}_{i j}\right)\right|\right),
\end{aligned}
$$


for some $\hat{\xi}_{i j} \in\left[\hat{x}_{i-1}, \hat{x}_{i+1}\right]$ and $\hat{\eta}_{i j} \in\left[\hat{y}_{j-1}, \hat{y}_{j+1}\right]$. We shall prove the analogue of estimate (5.9) only for the component $\psi_{1, i j}$ with $i \geq 0$, since for $\psi_{1, i j}$ with $i<0$, and for $\psi_{2, i j}$, analogous estimates are obtained similarly.

First, note that (5.5) and $\hat{\sigma} i^{3} / N^{3}=\hat{x}_{i} \leq \hat{r}_{i j}$ imply

$$
h_{i+1}-h_{i} \leq \frac{6 \hat{\sigma}^{2 / 3}}{N^{2}} \hat{r}_{i j}^{1 / 3}, \quad \hbar_{i} \leq h_{i+1} \leq C \frac{\hat{\sigma} i^{2}}{N^{3}} \leq C \frac{\hat{\sigma}^{1 / 3}}{N} \hat{r}_{i j}^{2 / 3} .
$$

Next, combining this with (5.10) and (5.2a), we get

$$
\left|\psi_{1, i j}\right| \leq C\left(\frac{\hat{\sigma}^{2 / 3}}{N^{2}} \hat{r}_{i j}^{1 / 3}\left[\hat{r}_{i j}^{2 / 3-3}+1\right]+\frac{\hat{\sigma}^{2 / 3}}{N^{2}} \hat{r}_{i j}^{4 / 3}\left[\hat{r}_{i-1, j}^{2 / 3-4}+1\right]\right) .
$$

Finally, noting that $|i|+|j| \geq 2$ implies $r_{i-1, j} \geq C r_{i j}$, and recalling (5.1) and $\hat{r}_{i j} \leq C \hat{\sigma}$, we obtain the desired estimate $\left|\psi_{1, i j}\right| \leq C N^{-2} \ln ^{2} N\left(\hat{r}_{i j}^{-2}+1\right)$.

Case B. $0<|i|+|j|<2$. Following the decomposition $(5.2 \mathrm{~b})$ of $\hat{u}$, we shall invoke the decomposition $\psi_{i j}:=\psi_{i j}[\hat{\chi}]+\psi_{i j}[\hat{z}]$, where $\psi_{i j}[\hat{\chi}]$ and $\psi_{i j}[\hat{z}]$ are defined similarly to $\psi_{i j}$ in (5.8) with $\hat{u}$ replaced by $\hat{\chi}$ and $\hat{z}$ respectively. Imitating the argument used for Case A, we immediately get $\left|\psi_{i j}[\hat{z}]\right| \leq C N^{-2} \ln ^{2} N$. Next, note that

$\left|\psi_{i j}[\hat{\chi}]\right| \leq C\left(h_{1}^{-2} \max _{|k|+|m| \leq 2}\left|\hat{\chi}\left(\hat{x}_{k}, \hat{y}_{m}\right)\right|+\left|\triangle \hat{\chi}\left(\hat{x}_{i}, \hat{y}_{j}\right)\right|\right) \leq C \hat{r}_{i j}^{-2}\left(\max _{|k|+|m| \leq 2} \hat{r}_{k m}^{2 / 3}+\hat{r}_{i j}^{2}\right)$,

since for $|i|+|j|<2$ we have $\hat{r}_{i j} \leq C h_{1}$, while $|\triangle \hat{\chi}|=|\triangle \hat{z}-q \hat{u}+\hat{f}| \leq C$. Finally, note that, by (5.3) and (5.1), we have $\hat{r}_{k m}^{2 / 3} \leq C\left(\hat{\sigma} / N^{3}\right)^{2 / 3} \leq C N^{-2} \ln ^{2} N$, which yields (5.9) in Case B.

5.2. Proof of Lemma 5.1, An analogue of Lemma 5.1 for the uniform mesh in a rectangular domain was presented by Volkov [21]; the proof that we present here closely imitates his argument.

Introduce the discrete Green's function $G_{i j ; i_{0} j_{0}}^{h}$ for the operator $\hat{L}^{h}$ in (5.4), which for each $\left(\hat{x}_{i_{0}}, \hat{y}_{j_{0}}\right) \in \hat{\Omega}_{\sigma}$, satisfies

$$
\begin{gathered}
\hat{L}^{h} G_{i j ; i_{0} j_{0}}^{h}=\delta^{h}\left(\hat{x}_{i}-\hat{x}_{i_{0}}, \hat{y}_{j}-\hat{y}_{j_{0}}\right), \quad\left(\hat{x}_{i}, \hat{y}_{j}\right) \in \hat{\Omega}_{\sigma} ; \\
G_{i j ; i_{0} j_{0}}^{h}=0, \quad\left(\hat{x}_{i}, \hat{y}_{j}\right) \in \partial \hat{\Omega}_{\sigma} .
\end{gathered}
$$

Here

$$
\delta^{h}\left(\hat{x}_{i}-\hat{x}_{i_{0}}, \hat{y}_{j}-\hat{y}_{j_{0}}\right)=\left\{\begin{array}{cl}
\hbar_{i_{0}}^{-1} \hbar_{j_{0}}^{-1}, & i=i_{0} \text { and } j=j_{0}, \\
0, & \text { otherwise, }
\end{array}\right.
$$

is a discrete analogue of the Dirac $\delta$-distribution.

The following version of a theorem from [15, 16] will be useful in our analysis.

Lemma 5.3 ([15, Theorem $3, \S 2$, Ch. IV]). Suppose $\mathcal{U}_{i j}=0$ for $\left(\hat{x}_{i}, \hat{y}_{j}\right) \in \partial \hat{\Omega}_{\sigma}$, and

$$
A_{i j} \mathcal{U}_{i j}-\sum_{\left(x_{k}, y_{m}\right) \neq\left(x_{i}, y_{j}\right)} B_{i j ; k m} \mathcal{U}_{k m}=F_{i j}, \quad\left(\hat{x}_{i}, \hat{y}_{j}\right) \in \hat{\Omega}_{\sigma}
$$

where

$$
B_{i j, k m} \geq 0, \quad D_{i j}:=A_{i j}-\sum_{\left(x_{k}, y_{m}\right) \neq\left(x_{i}, y_{j}\right)} B_{i j, k m}>0 .
$$

Then

$$
\max _{i j}\left|\mathcal{U}_{i j}\right| \leq \max _{i j}\left|\frac{F_{i j}}{D_{i j}}\right|
$$


We shall invoke the above lemma to obtain the following estimate for the discrete Green's function.

Lemma 5.4. Fix arbitrary $\bar{C}>0$ and let $\left|i_{0}\right|+\left|j_{0}\right| \leq \bar{C}$. Then

$$
0 \leq G_{i j ; i_{0} j_{0}}^{h} \leq C \quad \text { for all }\left(\hat{x}_{i}, \hat{y}_{j}\right) \in \hat{\Omega}_{\sigma} .
$$

Proof. The lower bound in (5.13) immediately follows from the discrete maximum principle. Now we shall obtain the upper bound. To simplify the presentation, within this proof we shall fix $i_{0}, j_{0}$ and use the notation $G_{i j}^{h}:=G_{i j ; i_{0} j_{0}}^{h}$. Note that our argument will involve the values $h_{i}$ and $h_{j}$ for $|i|+|j| \leq \bar{C}+1$, for which, by (5.5), we have

$$
h_{1} \leq h_{i} \leq C h_{1}, \quad h_{1} \leq h_{j} \leq C h_{1} .
$$

(i) We start with a particular case of $i_{0}=-1,0 \leq j_{0}<\bar{C}$. Rewriting the discrete equation (5.11a) in the form (5.12) and noting that (5.11b) implies $G_{0, j_{0}}^{h}=0$, we observe that

$$
D_{-1, j_{0}}=\hat{q}\left(\hat{x}_{-1}, \hat{y}_{j_{0}}\right)+\frac{1}{h_{0} \hbar_{-1}}, \quad F_{-1, j_{0}}=\hbar_{-1}^{-1} \hbar_{j_{0}}^{-1},
$$

while at the other interior mesh nodes we have $D_{i j} \geq \hat{q}\left(\hat{x}_{i}, \hat{y}_{j}\right)$ and $F_{i j}=0$. Now, Lemma 5.3 implies that $G_{i j}^{h} \leq F_{-1, j_{0}} / D_{-1, j_{0}}$, which, by (5.14), yields the upper estimate in (5.13).

Note that for $j_{0}=-1,0 \leq i_{0}<\bar{C}$, estimate (5.13) can be established similarly.

(ii) Next, consider $i_{0}=-2,0 \leq j_{0}<\bar{C}$. Now $D_{-1, j_{0}}$ is given by the same formula from (5.15), while $F_{-1, j_{0}}=0$. We shall focus on the discrete equation at $\left(\hat{x}_{-2}, \hat{y}_{j_{0}}\right)$, where $D_{-2, j_{0}}=\hat{q}\left(\hat{x}_{-2}, \hat{y}_{j_{0}}\right)$ and $F_{-2, j_{0}}=\hbar_{-2}^{-1} \hbar_{j_{0}}^{-1}$. Modify this equation by combining it with the equation at $\left(\hat{x}_{-1}, \hat{y}_{j_{0}}\right)$ eliminating $G_{-1, j_{0}}^{h}$ :

$$
\text { new eq. at }\left(-2, j_{0}\right):=\text { eq. at }\left(-2, j_{0}\right)+\frac{B_{-2, j_{0} ;-1, j_{0}}}{A_{-1, j_{0}}} \text { eq. at }\left(-1, j_{0}\right) \text {. }
$$

Furthermore, since $D_{i j}$ is the sum of the coefficients, we observe that

$$
\tilde{D}_{-2, j_{0}}:=D_{-2, j_{0}}+\frac{B_{-2, j_{0} ;-1, j_{0}}}{A_{-1, j_{0}}} D_{-1, j_{0}}, \quad \tilde{F}_{-2, j_{0}}=F_{-2, j_{0}}=\hbar_{-2}^{-1} \hbar_{j_{0}}^{-1},
$$

where we used the notation $\tilde{D}_{-2, j_{0}}$ and $\tilde{F}_{-2, j_{0}}$ for the quantities $D_{i j}$ and $F_{i j}$ in the new equation. Note that

$$
B_{-2, j_{0} ;-1, j_{0}}=\frac{1}{h_{-1} \hbar_{-2}}, \quad D_{-1, j_{0}}<A_{-1, j_{0}}<4 D_{-1, j_{0}},
$$

which implies that $\tilde{D}_{-2, j_{0}} \geq \hat{q}\left(\hat{x}_{-2}, \hat{y}_{j_{0}}\right)+\frac{1}{4 h_{-1} \hbar_{-2}}$. Now, applying Lemma 5.3, we get $G_{i j}^{h} \leq \tilde{F}_{-2, j_{0}} / \tilde{D}_{-2, j_{0}}$, which, by (15.14), again yields the upper estimate in (5.13).

Similarly, one can establish estimate (5.13) for $i_{0}=j_{0}=-1$ and for $j_{0}=-2$, $0 \leq i_{0}<\bar{C}$.

(iii) We continue repeating this argument; although as we move away from the boundary $\partial \hat{\Omega}_{\sigma}$, the constant multiplier in (5.13) increases; nevetherless, the argument can be applied a fixed number of times.

Corollary 5.5. Fix arbitrary $\bar{C}>0$ and let $B_{i j}=0$ for $\left(\hat{x}_{i}, \hat{y}_{j}\right) \in \partial \hat{\Omega}_{\sigma}$,

$$
\hat{L}^{h} B_{i j}=\left\{\begin{array}{cl}
\hat{r}_{i j}^{-2}+1, & |i|+|j| \leq \bar{C}, \\
0, & \text { otherwise, }
\end{array} \quad \text { for } \quad\left(\hat{x}_{i}, \hat{y}_{j}\right) \in \hat{\Omega}_{\sigma} .\right.
$$


Then $0 \leq B_{i j} \leq C$.

Proof. This follows from the representation of $B_{i j}$ through the discrete Green's function

$$
B_{i j}=\sum_{\left|i_{0}\right|+\left|j_{0}\right| \leq \bar{C}} G_{i j ; i_{0} j_{0}}^{h}\left(\hat{L}^{h} B_{i_{0} j_{0}}\right) \hbar_{i_{0}} \hbar_{j_{0}},
$$

since this sum involves a finite number of terms and each of them is $O(1)$.

Proof of Lemma 5.1. Introduce a barrier function

$$
\Phi(\hat{x}, \hat{y}):=(3 \pi-\varphi) \varphi,
$$

where we use the standard polar coordinates $(\hat{r}, \varphi)$ of the point $(\hat{x}, \hat{y}) \in \hat{\Omega}_{\sigma}$. Since our domain corresponds to $\varphi \in[\pi / 2,2 \pi]$, clearly we have $\Phi \geq 5 \pi^{2} / 4$. Furthermore, since $\triangle \Phi=\hat{r}^{-2} \Phi_{\varphi \varphi}$, we get

$$
\hat{L} \Phi=2 \hat{r}^{-2}+\hat{q} \Phi \geq C\left(\hat{r}^{-2}+1\right) .
$$

We claim that for some sufficiently large $\bar{C}$ we have

$$
\hat{L}^{h} \Phi\left(\hat{x}_{i}, \hat{y}_{j}\right) \geq\left\{\begin{array}{rr}
C_{1}\left(\hat{r}_{i j}^{-2}+1\right), & |i|+|j|>\bar{C}, \\
-C_{2}\left(\hat{r}_{i j}^{-2}+1\right), & |i|+|j| \leq \bar{C} .
\end{array}\right.
$$

Then, using $B_{i j}$ from Corollary 5.5, we construct the barrier

$$
\tilde{\Phi}_{i j}:=\Phi\left(\hat{x}_{i}, \hat{y}_{j}\right)+\left(C_{1}+C_{2}\right) B_{i j}
$$

so that $\hat{L}^{h} \tilde{\Phi}_{i j} \geq C_{1}\left(\hat{r}_{i j}^{-2}+1\right)$. Now, applying the discrete comparison principle to problem (5.6), we obtain $\left|\phi_{i j}\right| \leq C \tilde{\Phi}_{i j}$, which yields $\left|\phi_{i j}\right| \leq C$.

Thus, to complete the proof, it remains to establish (5.17). Consider two cases.

Case A. $|i|+|j|>\bar{C}$ for some sufficiently large $\bar{C}$. Using Taylor series expansions, we observe that

$$
\left|\hat{L}^{h} \Phi\left(\hat{x}_{i}, \hat{y}_{j}\right)-\hat{L} \Phi\left(\hat{x}_{i}, \hat{y}_{j}\right)\right| \leq C\left(\hbar_{i}\left|\Phi^{(3,0)}\left(\hat{\xi}_{i j}, \hat{y}_{j}\right)\right|+\hbar_{j}\left|\Phi^{(0,3)}\left(\hat{x}_{i}, \hat{\eta}_{i j}\right)\right|\right)
$$

for some $\hat{\xi}_{i j} \in\left[\hat{x}_{i-1}, \hat{x}_{i+1}\right]$ and $\hat{\eta}_{i j} \in\left[\hat{y}_{j-1}, \hat{y}_{j+1}\right]$. Note that $\left|\Phi^{(3,0)}\right|+\left|\Phi^{(0,3)}\right| \leq C \hat{r}^{-3}$ since

$$
\left[\begin{array}{c}
\frac{\partial}{\partial \hat{x}} \\
\frac{\partial}{\partial \hat{y}}
\end{array}\right]=\left[\begin{array}{rr}
\cos \varphi & -\sin \varphi \\
\sin \varphi & \cos \varphi
\end{array}\right]\left[\begin{array}{c}
\frac{\partial}{\partial \hat{r}} \\
\frac{1}{\hat{r}} \frac{\partial}{\partial \varphi}
\end{array}\right] .
$$

Furthermore, $|i|+|j|>\bar{C}$ implies $\hat{r}_{i \pm 1, j} \geq C \hat{r}_{i j}$ and $\hat{r}_{i, j \pm 1} \geq C \hat{r}_{i j}$, and also $\hbar_{i}+\hbar_{j} \leq C_{0} \hat{r}_{i j}$. Hence

$$
\left|\hat{L}^{h} \Phi\left(\hat{x}_{i}, \hat{y}_{j}\right)-\hat{L} \Phi\left(\hat{x}_{i}, \hat{y}_{j}\right)\right| \leq C\left(\hbar_{i}+\hbar_{j}\right) \hat{r}_{i j}^{-3} \leq C C_{0} \hat{r}_{i j}^{-2} .
$$

Note that choosing $\bar{C}$ sufficiently large, we can make the constant $C_{0}$ arbitrarily small so that (5.16) combined with (5.18) yields (5.17) in Case A.

Case B. $|i|+|j| \leq \bar{C}$. Now we have $\hat{r}_{i j} \leq C h_{1}$ and (5.14), and hence

$$
\left|\hat{L}^{h} \Phi\left(\hat{x}_{i}, \hat{y}_{j}\right)\right| \leq C h_{1}^{-2} \max _{|i|+|j| \leq \bar{C}} \Phi_{i j} \leq C \hat{r}_{i j}^{-2},
$$

which yields (5.17) in Case B. 
TABLE 1. Maximum nodal values of double mesh errors $E^{N}$ and computational rates $r$ in $\left(N^{-1} \ln N\right)^{r}$

\begin{tabular}{cccccc}
\hline$N$ & $\varepsilon=1$ & $\varepsilon=10^{-1}$ & $\varepsilon=10^{-2}$ & $\varepsilon=10^{-4}$ & $\varepsilon=10^{-8}$ \\
\hline 12 & $1.57 \mathrm{e}-4$ & $2.60 \mathrm{e}-3$ & $5.19 \mathrm{e}-3$ & $4.96 \mathrm{e}-3$ & $4.95 \mathrm{e}-3$ \\
24 & $4.16 \mathrm{e}-5$ & $6.62 \mathrm{e}-4$ & $1.90 \mathrm{e}-3$ & $1.90 \mathrm{e}-3$ & $1.90 \mathrm{e}-3$ \\
48 & $1.07 \mathrm{e}-5$ & $1.65 \mathrm{e}-4$ & $7.19 \mathrm{e}-4$ & $7.19 \mathrm{e}-4$ & $7.19 \mathrm{e}-4$ \\
96 & $2.73 \mathrm{e}-6$ & $4.41 \mathrm{e}-5$ & $2.52 \mathrm{e}-4$ & $2.52 \mathrm{e}-4$ & $2.52 \mathrm{e}-4$ \\
\hline 12 & 2.97 & 3.06 & 2.25 & 2.14 & 2.14 \\
24 & 2.73 & 2.80 & 1.96 & 1.96 & 1.96 \\
48 & 2.59 & 2.50 & 1.98 & 1.98 & 1.98 \\
\hline
\end{tabular}

\section{Numerical RESUlts}

Our test problem is (1.1) with

$$
\begin{gathered}
q \equiv 1, \quad f=2 /\left(2+x^{2}-x y\right), \quad g_{2}=-y^{2} / 2, \quad g_{3}=-(1+x) / 2, \\
g_{k} \equiv 0, \quad k=1,4,5,6 ;
\end{gathered}
$$

see Figure 1.

Table 1 presents numerical results for our method with the parameter $\alpha:=(1.1)^{-1}$, which is used in (1.4) to compute the mesh transition parameter $\sigma$. Since the exact solution is unknown, to investigate the rates of convergence, for each $N$ and $\varepsilon$, we compute the double mesh errors $E^{N}:=\max _{i j}\left|U_{i j}^{N}-\bar{U}_{2 i, 2 j}^{2 N}\right|$. Here $U^{N}:=U$ is the computed solution on the mesh described in $§ 1$ with $6 N+1$ mesh nodes in each direction, while $\bar{U}^{2 N}$ is the computed solution on a similar mesh that uses the same transition parameter $\sigma$, but the (almost) doubled number $6(2 N)+1$ of mesh nodes in each direction. The table also shows the rates of convergence in $\left(N^{-1} \ln N\right)^{r}$, computed as described, e.g., in [11, §4.2], by

$$
r:=\frac{\log _{2}\left(E^{N} / E^{2 N}\right)}{\log _{2} \frac{2 \log _{2} N}{\log _{2} N+1}} .
$$

We observe that the double mesh errors $E^{N}$ stabilize as $\varepsilon \rightarrow 0$, while the computational rates of convergence in $\left(N^{-1} \ln N\right)^{r}$ are quite close to 2 for $\varepsilon \ll 1$ and even higher for $\varepsilon=1$, for which one can prove that the errors are $O\left(N^{-2}\right)$. In summary, the numerical results fully agree with our error estimate of Theorem 3.1 .

\section{REFERENCES}

[1] V. B. Andreev, Grid approximations of nonsmooth solutions of differential equations, Differ. Uravn., 16 (1980), no. 7, 1172-1184 (Russian). MR581768 (82g:65051)

[2] V. B. Andreev, On the accuracy of grid approximations for nonsmooth solutions of singularly perturbed reaction-diffusion equations in a square region, Differ. Uravn., 42 (2006), no. 7, 895906 (Russian), translation in Differential Equations, 42 (2006), no. 7, 954-966. MR.2294140

[3] V. B. Andreev, Uniform grid approximation for nonsmooth solutions of a mixed problem for a singularly perturbed reaction-diffusion equation in a rectangular domain, in preparation.

[4] I.A. Blatov, Galerkin finite element method for elliptic quasilinear singularly perturbed boundary problems. I, Differ. Uravn., 28 (1992), 1168-1177 (Russian), translation in Differ. Equ., 28 (1992), 931-940. MR1201213 (94a:65056) 
[5] C. Clavero, J. L. Gracia, E. O'Riordan, A parameter robust numerical method for a two dimensional reaction-diffusion problem, Math. Comp., 74 (2005), 1743-1758. MR2164094 (2006e:65192)

[6] G. M. Fichtenholz, Differential and integral calculus, Vol. I, Fizmatgiz, Moscow, 1962.

[7] P. Grisvard, Elliptic problems in nonsmooth domains, Pitman, Boston, 1985. MR775683 (86m:35044)

[8] R. B. Kellogg, Corner singularities and singular perturbations, Ann. Univ. Ferrara, Sez. VII, 47 (2001), 177-206. MR.1897566 (2003c:35031)

[9] V. A. Kondrat'ev, Boundary value problems for elliptic equations in domains with conical or angular points, Trudy Moskov. Mat. Obšč., 16 (1967), 209-292 (Russian), translation in Trans. Moscow Math. Soc., 16 (1967), 227-313. MR0226187 (37:1777)

[10] N. Kopteva, Maximum norm error analysis of a $2 d$ singularly perturbed semilinear reactiondiffusion problem, Math. Comp., 76 (2007), 631-646. MR2291831

[11] N. Kopteva and M. Stynes, Numerical analysis of a singularly perturbed nonlinear reactiondiffusion problem with multiple solutions, Appl. Numer. Math., 51 (2004), 273-288. MR2091404 (2005e:65097)

[12] O. A. Ladyzhenskaya and N. N. Ural'tseva, Linear and quasilinear elliptic equations, Academic Press, New York, 1968. MR0244627 (39:5941)

[13] J.M. Melenk, hp-finite element methods for singular perturbations, Springer, 2002. MR.1939620 (2003i:65108)

[14] H.-G. Roos, M. Stynes and L. Tobiska, Numerical methods for singularly perturbed differential equations, Springer-Verlag, Berlin, 1996. MR.1477665 (99a:65134)

[15] A. A. Samarskii, Theory of difference schemes, Nauka, Moscow, 1989 (Russian), translation in A. A. Samarskii, The theory of difference schemes, Marcel Dekker, New York, 2001. MR.1196231 (93g:65004)

[16] A. A. Samarski and V. B. Andreev, Difference methods for elliptic equations, Nauka, Moscow, 1976 (Russian). MR0502017 (58:19209a)

[17] A.H. Schatz and L.B. Wahlbin, On the finite element method for singularly perturbed reaction-diffusion problems in two and one dimensions, Math. Comp., 40 (1983), 47-89. MR679434 (84c:65137)

[18] G. I. Shishkin, Grid approximation of singularly perturbed elliptic and parabolic equations, Ur. O. Ran, Ekaterinburg, 1992 (Russian).

[19] G. I. Shishkin, Grid approximation of singularly perturbed boundary value problems in a nonconvex domain with a piecewise-smooth boundary, Mat. Model., 11 (1999), no. 11, 75-90 (Russian). MR1761699 (2001g:65133)

[20] E. A. Volkov, Differential properties of solutions of boundary value problems for the Laplace equation on polygons, Trudy Mat. Inst. Steklov, 77 (1965), 113-142 (Russian), translation in Proc. Steklov Inst. Math., 77 (1965), 127-159. MR0192078 (33:305)

[21] E. A. Volkov, The method of composite regular nets for the Laplace equation on polygons, Trudy Mat. Inst. Steklov, 140 (1976), 68-102 (Russian), translation in Proc. Steklov Inst. Math., 140 (1979), 71-109. MR0448945 (56:7250)

Faculty of Computational Mathematics and Cybernetics, Moscow State University, Leninskie Gory, 119991, Moscow, Russia

E-mail address: andreev@cs.msu.su

Department of Mathematics and Statistics, University of Limerick, Limerick, Ireland E-mail address: natalia.kopteva@ul.ie 